\title{
Full-Discrete Weak Galerkin Finite Element Method for Solving Diffusion- Convection Problem.
}

\author{
Hashim A. Kashkool and Asmaa H. Aneed \\ Dep. of Mathematics, College of Education University of Basrah, Basrah, Iraq. \\ hkashkool@yahoo.com \\ asmaahammdan93@gmail.com
}

\begin{abstract}
This paper applied and analyzes full-discrete weak Galerkin (WG) finite element method for non steady two dimensional convection-diffusion problems on conforming polygon. We approximate the time derivative by backward finite difference method and the elliptic form by WG finite element method. The main idea of WG finite element methods is the use of weak functions and their corresponding discrete weak derivatives in standard weak form of the model problem. The theoretical evidence proved that the error estimate in $L^{2}$-norm, the properties of the bilinear form $a(u, v)$, (v-elliptic and continuity), stability, and the energy conservation law.
\end{abstract}

\section{Keywords}

Convection -diffusion equation; full discrete weak Galerkin finite element method; error estimate; conservation law.

\section{AMS subject classifications: $65 \mathrm{M} 60,65 \mathrm{M} 30$.}

\section{Introduction:}

Weak Galerkin (WG) finite element methods refer to general finite element methods for partial differential equations (PDEs). The novel idea of WG finite element methods is on the use of weak functions, weak gradients and their approximations results in a new concept called discrete weak gradients which is expected to play important roles in numerical methods for PDEs [6]. The key to WG finite element methods is the use of a discrete weak gradients operator which is defined and computed by solving inexpensive problems locally on each element. The fundamental difference between the weak Galerkin finite element method and other existing methods, such as Discontinuous Galerkin Finite Element Method ([1, 3, 17]) and the Finite Volume Method $([4,7,18])$, is used of weak function and weak gradient in the design of numerical scheme based on existing weak forms for the underlying PDEs.

WG finite element methods were first introduced in [6] for solving steady second order elliptic problem and later on in [8] for shape regular polytopal meshes. The method has been successfully applied to elliptic interface problems [10], Helmholtz equations [13], and biharmonic equations [11, 12], in this paper, we applied the WG finite element methods for non steady diffusion convection problem and proved that the error estimate in $L^{2}$-norm, the elliptic property, and the energy conservation law. This paper is organized as follows: Section 2 is devoted to a description some preliminaries and notations, In section 3 we show the Convection-Diffusion Problem. The weak function the weak gradient and the weak Galerkin method. In section 4 we discuses the full-discrete weak Galerkin method. In section 5 we proved the energy conservation law of WG finite element method. The error analysis for Semi- discrete weak Galerkin finite element method proved in section 6 .

\section{Preliminaries and Notations:}

Let $D$ be any open and bounded domain in $R^{d}(d=2)$. We use the standard definition for the Sobolev space $H^{s}(D)$ and their associated inner products $(\cdot,)_{s, D}$ norms \|\|$_{s, D}$, and semi-norms $\|_{s, D}$ for any $s \geq 0[5,14]$. For example, for any integer $s \geq 0$, the semi-norm $\|_{s, D}$ is given by

$$
|u|_{s, D}=\left(\sum_{|\alpha|=s_{D}} \int_{\mid}\left|\partial^{\alpha} u\right|^{2} d D\right)^{\frac{1}{2}},
$$

With the usual notation,

$$
\alpha=\left(\alpha_{1}, \alpha_{2}\right),|\alpha|=\alpha_{1}+\alpha_{2}, \quad \partial^{\alpha}=\partial_{x_{1}}^{\alpha_{1}} \partial_{x_{2}}^{\alpha_{2}},
$$

The Sobolev norm \|\|$_{s, D}$ is given by, 


$$
\|u\|_{s, D}=\left(\sum_{j=0}^{s}|u|_{j, D}^{2}\right)^{\frac{1}{2}}
$$

The space $H^{s}(D)$ coincides with $L^{2}(D)$, for which the norm and the inner product are denoted by \|\|$_{D}$, and $(\cdot,)_{D}$ respectively. When $D=\Omega$, we shall drop the Subscript $D$ in the norm and inner product notation. The space $H(\operatorname{div}, D)$ is defined as the set of vector-valued functions on $D$ which, together with their divergence, are square integrable, i.e.,

$$
H(\operatorname{div}, D)=\left\{u: u \in\left[L^{2}(D)\right]^{d}, \nabla \cdot u \in L^{2}(D)\right\},
$$

The norm in $H(\operatorname{div}, D)$ is defined by,

$$
\|u\|_{\mathrm{H}(\mathrm{div} ; \mathrm{D})}=\left(\|u\|_{D}^{2}+\|\nabla \cdot u\|_{D}^{2}\right)^{\frac{1}{2}}
$$

Definition 2.1 (Bochner Space [1]): Let $X$ be a Banach space with a norm \|\|$_{X}$ and a semi- norm $\left.\right|_{X}$. Then define:

$$
\begin{aligned}
& C([0, T] ; X)=\left\{v:[0, T] \rightarrow X, \text { continuous },\|v\|_{C([0, T] ; X)}=\sup _{t \in[0, T]}\|v\|_{X}<\infty\right\} \\
& L^{2}(0, T ; X)=\left\{v:(0, T) \rightarrow \text {, stronglymeasurable }\|v\|_{L^{2}(0, T ; X)}^{2}=\int_{0}^{T}\|v\|_{X}^{2} d t<\infty\right\},
\end{aligned}
$$

And

$$
H^{r}(0, T ; X)=\left\{v \in L^{2}(0, T ; X) ;\|v\|_{H^{r}(0, T ; X)}^{2}=\int_{0}^{T} \sum_{\alpha=0}^{r}\left\|\frac{\partial^{\alpha} v}{\partial t^{\alpha}}\right\|_{X}^{2} d t<\infty\right\}
$$

Moreover ,

$$
|v|_{C([0, T] ; X)}=\sup _{t \in[0, T]}\|v\|_{X},|v|_{L^{2}(0, T ; X)}^{2}=\left(\int_{0}^{T}\|v\|_{X}^{2} d t\right)^{\frac{1}{2}},
$$

Definition 2.2 [2]: Young's inequality ( $\varepsilon$-inequality) is defined by

$$
a b \leq \frac{1}{2 \varepsilon} a^{2}+\frac{\varepsilon}{2} b^{2}
$$

for $a$ and $b$ are real numbers and $\varepsilon \geq 0$.

Definition 2.3 [15]: In a Hilbert space $V$ the Cauchy-Schwartz inequality which is defined by,

$$
|(u, v)| \leq\|u\|\|v\|,
$$

for each $u$ and $v \in V$.

\section{The Convection-Diffusion Problem:}

We consider the non- state diffusion-convection problem,

$$
\frac{\partial u}{\partial t}-\lambda \Delta u+b \cdot \nabla u=f, \quad \Omega \times(0, T)=Q_{T}
$$

Subject to

$$
u \mid \partial \Omega \times(0, T)=u_{D}, \text { on } \partial \Omega \text { and } u(x, 0)=u^{0}(x) \quad x \in \Omega
$$


In a domain $\Omega \subset R^{2}$ with boundary $\partial \Omega$, where $x=\left(x_{1}, x_{2}\right)$ and $f=(x, t)$, where $\Omega$ a polygonal domain in $R^{2}, \nabla u$ denotes the gradient of the function $u=u(x)$, and $\nabla$ is known as the gradient operator. And $\lambda$ is diffusion coefficient, $b=\left(b_{1}, b_{2}\right)$ is a velocity vector.

For simplicity, let the function $f$ in (1) be locally integrable in $\Omega$. We shall consider solutions of (1) with a nonhomogeneous Dirichlet boundary condition $u(0)=u_{D}$, where $u_{D} \in H^{\frac{1}{2}}(\partial \Omega)$ is a function defined on the boundary of $\Omega$. Here $H^{1}(\Omega)$ is the Sobolev space consisting of functions which, together with their gradients, are square integrable over $\Omega, H^{\frac{1}{2}}(\partial \Omega)$ is the trace of $H^{1}(\Omega)$ on the boundary of $\Omega$, The standard weak form for seeks $u \in H^{1}(\Omega)$. Such that $u=u_{D}$ on $\partial \Omega$.

$$
\left(u_{t}, v\right)+(\lambda \nabla u, \nabla v)+(b u, \nabla v)=(f, v), \quad \forall v \in H_{0}^{1}(\Omega)
$$

\subsection{THE WEAK GRADIENT:}

The main idea of weak Galerkin methods is the use of discrete weak derivatives in the place of strong derivatives in the variational form for the underlying partial differential equations. The weak gradient operator will then be employed to discretization the problem (3) through the use of a discrete weak gradient operator as building bricks [6]. The discrete weak gradient is given by approximating the weak gradient operator with piecewise polynomial functions. To introduced the weak gradient operator;

Firstly we define the weak function. Let $K$ be any polygonal domain with interior $K^{0}$ and boundary $\partial K$. A weak function on the region $K$ refers to a vector-valued function $v=\left\{v_{0}, v_{b}\right\}$ such that $v_{0} \in L^{2}(K)$ and $v_{b} \in H^{\frac{1}{2}}(\partial K)$. The first component $v_{0}$ can be understood as the value of $v$ in the interior of $K$, and the second component $v_{b}$ represents $v$ on the boundary of $K$. Denote by $W(K)$ the space of weak functions on $K$ [9]; i.e.

$$
W(K)=\left\{v=\left\{v_{0}, v_{b}\right\}, v_{0} \in L^{2}(K), v_{b} \in H^{\frac{1}{2}}(\partial K)\right\} .
$$

Secondly, we define the weak gradient, for any $v \in W(K)$, the weak gradient of $v$ is defined as a linear functional $\nabla$ w in the dual space of $H(d i v ; K)$ whose action on each $q \in H(\operatorname{div}, K)$ is given by

$$
\left(\nabla_{w} v, q\right)_{k}=-\int_{K} v_{0} \nabla \cdot q d K+\int_{\partial K} v_{b} q \cdot n d s,
$$

where $n$ is the outward normal direction to $\partial K$.

The discrete weak gradient operator was defined by approximating $\nabla_{w} v$ in a polynomial subspace of the dual of $H(\operatorname{div}, K)$. More precisely, for any non-negative integer $r \geq 0$, denote by $P_{r}(K)$ the set of polynomials on $K$ with degree no more than $r$. Let $V(K, r) \subset\left[P_{r}(K)\right]^{2}$ be a subspace of the space of vector-valued polynomials of degree $r$. The discrete weak gradient operator, denoted by $\nabla_{d}$ is defined as the unique polynomial $\nabla_{d} v \in V(K, r) \subset H(\operatorname{div}, K)$ satisfying the following equation:

$$
\left(\nabla_{d} v, q\right)=-\int_{K} v_{0} \nabla \cdot q d K+\int_{\partial K} v_{b} q \cdot n d s, \quad \forall q \in V(K, r) .
$$

\subsection{WEAK GALERKIN FINITE ELEMENT SPACE:}

Let $T_{h}$ be a triangular partition of the domain $\Omega$ with $\partial T$ as its boundary. For each $T \in T_{h}$, Denote by $P_{j}\left(T^{0}\right)$ the set of polynomials on $T^{0}$ with degree no more than $j$, and $P_{\ell}(\partial T)$ the set of polynomials on $\partial T$ with degree no more than $\ell$. A discrete weak function $v=\left\{v_{0}, v_{b}\right\}$ on $T$ refers to a weak function $v=\left\{v_{0}, v_{b}\right\}$ such that $v_{0} \in P_{j}\left(T^{0}\right)$ and $v_{b} \in P_{\ell}(\partial T)$ with $j \geq 0$ and $\ell \geq 0$. Denote this space by $W(T, j, \ell)$,i.e.,

$$
W(T, j, \ell)=\left\{v=\left\{v_{0}, v_{b}\right\}: v_{0} \in p_{j}\left(T^{0}\right), v_{b} \in P_{\ell}(\partial T)\right\} .
$$

The corresponding finite element space would be defined by patching $W(T, j, \ell)$ over all the triangles $T \in T_{h}$. In other words; the weak finite element space is given by 


$$
S_{h}(j, \ell)=\left\{v=\left\{v_{0}, v_{b}\right\}:\left.\left\{v_{0}, v_{b}\right\}\right|_{T} \in W(T, j, \ell), \forall T \in T_{h}\right\}
$$

Denote by $S_{h}^{0}(j, \ell)$ the subspace of $S_{h}(j, \ell)$ with vanishing boundary values on $\partial \Omega$; i.e.

$$
S_{h}^{0}(j, \ell)=\left\{v=\left\{v_{0}, v_{b}\right\} \in S_{h}(j, \ell),\left.v_{b}\right|_{\partial T \cap \partial \Omega}=0, \forall T \in T_{h}\right\}
$$

According to (6) for each $v=\left\{v_{0}, v_{b}\right\} \in S_{h}(j, \ell)$, the discrete weak gradient of $v$, on each element $T$ is given by the following equation:

$$
\left(\nabla_{d} v, q\right)_{T}=-\int_{T} v_{0} \nabla \cdot q d T+\int_{\partial T} v_{b} q \cdot n d s, \quad \forall q \in V(T, r)
$$

For each element $T \in T_{h}$, denote by $Q_{0}$ the $L^{2}$-projection from $L^{2}(T)$ to $P_{j}\left(T^{0}\right)$ and by $Q_{b}$ the $L^{2}(T)$ projection from $H^{\frac{1}{2}}(\partial T)$ to $P_{\ell}(\partial T)$. Denote by $R_{h}$ the $L^{2}$-projection of $\left[L^{2}(T)\right]^{2}$ onto the local discrete gradient space $\left[P_{r}(K)\right]^{2}$. Let $V=H^{1}(\Omega)$.We define a projection operator $Q_{h}: V \rightarrow S_{h}(j, \ell)$ so that on each element $T \in T_{h}$

$$
Q_{h} v=\left\{Q_{0} v, Q_{b} v\right\}, \quad \forall v=\left\{v_{0}, v_{b}\right\} \in W(T, j, \ell) .
$$

Lemma 3.2.1: Let $Q_{h}$ and $R_{h}$ be the $L^{2}$-projection operator defined in previous sections. Then, on each element $T \in T_{h}$, we have the following commutative property

$$
\nabla_{d}\left(Q_{h} u\right)=R_{h}(\nabla u), \quad \forall u \in H^{1}(T)
$$

Proof: According to the definition of $\nabla_{d}$, the discrete weak gradient function $\nabla_{d}\left(Q_{h} u\right)$ is given by the following equation:

$$
\left(\nabla_{d}\left(Q_{h} u\right), q\right)_{T}=-\int_{T}\left(Q_{0} u\right) \nabla \cdot q d T+\int_{\partial T}\left(Q_{b} u\right) q \cdot n d s, \quad \forall q \in V(T, r) .
$$

Since $Q_{0}$ and $Q_{b}$ are $L^{2}$-projection operators, then the right-hand side of (12) is given by

$$
\begin{aligned}
-\int_{T}\left(Q_{0} u\right) \nabla \cdot q d T+\int_{\partial T}\left(Q_{b} u\right) q \cdot n d s & =-\int_{T} u \nabla \cdot q d T+\int_{\partial T} u q \cdot n d s \\
& =\int_{T}(\nabla u) \cdot q d T=\int_{T} R_{h}(\nabla u) \cdot q d T
\end{aligned}
$$

This implies the desired identity (11).

One can also define the projection $\pi_{h}$ be the usual projection operator in the Dirichlet finite element method such that $\pi_{h} q \in H(T, r)$, and on each $T \in T_{h}$, one has $\pi_{h} q \in V(T, r)$ and satisfying.

$$
\left(\nabla \cdot q, v_{0}\right)_{T}=\left(\pi_{h} q, \nabla_{w} v\right)_{T}, \quad \forall v_{0} \in p_{j}\left(T^{0}\right)
$$

The following result is based on the above property of $\pi_{h}$.

Lemma 3.2.2 [6]: For any $q \in H(\operatorname{div}, K)$, we have

$$
\sum_{T \in T_{h}}\left(\nabla \cdot q, v_{0}\right)_{T}=\sum_{T \in T_{h}}\left(\pi_{h} q, \nabla_{w} v\right)_{T}, \quad \forall v=\left\{v_{0}, v_{b}\right\} \in S_{h}^{0}(j, \ell) .
$$

For any $u, v \in S_{h}(j, \ell)$ we introduce the following bilinear form

$$
a(u, v)=\left(\lambda \nabla_{d} u, \nabla_{d} v\right)-\left(b u_{0}, \nabla_{d} v\right),
$$

where 


$$
\begin{gathered}
\left(\lambda \nabla_{d} u, \nabla_{d} v\right)=\int_{\Omega} \lambda \nabla_{d} u \cdot \nabla_{d} v d \Omega \\
\left(b u_{0}, \nabla_{d} v\right)=\int_{\Omega} b u_{0} \cdot \nabla_{d} v d \Omega .
\end{gathered}
$$

\section{FULL-DISCRETE WEAK GALERKIN FINITE ELEMENT METHOD [15]:}

We now turn our attention to some discrete time weak Galerkin procedure. We introduce a time step $k$ and the time levels $t=t_{n}=n k$. Where $n$ is a nonnegative integer, and denote by $U^{n}=U_{h}^{n} \in S_{h}(j, \ell)$ the approximation of $u\left(t_{n}\right)$ to be determined. The backward Euler weak Galerkin method is define by a backward difference equation , if $\bar{\partial} U_{h}^{n}=\frac{\left(U_{h}^{n}-U_{h}^{n-1}\right)}{k}$

$$
\begin{aligned}
& \left(\bar{\partial} U_{h}^{n}, v_{0}\right)+\left(\lambda \nabla_{d} U_{h}^{n}, \nabla_{d} v\right)-\left(b U_{0}^{n}, \nabla_{d} v\right)=\left(f\left(t_{n}\right), v_{0}\right), \quad \forall v=\left\{v_{0}, v_{b}\right\} \in S_{h}^{0}(j, \ell) \\
& \left(\frac{\left(U_{h}^{n}-U_{h}^{n-1}\right)}{k}, v_{0}\right)+\left(\lambda \nabla_{d} U_{h}^{n}, \nabla_{d} v\right)-\left(b U_{0}^{n}, \nabla_{d} v\right)=\left(f\left(t_{n}\right), v_{0}\right) \\
& \left(U_{h}^{n}, v_{0}\right)-\left(U_{h}^{n-1}, v_{0}\right)+k\left(\lambda \nabla_{d} U_{h}^{n}, \nabla_{d} v\right)-k\left(b U_{0}^{n}, \nabla_{d} v\right)=k\left(f\left(t_{n}\right), v_{0}\right) \\
& \left(U_{h}^{n}, v_{0}\right)+k\left(\lambda \nabla_{d} U_{h}^{n}, \nabla_{d} v\right)-k\left(b U_{0}^{n}, \nabla_{d} v\right)=\left(U_{h}^{n-1}+k f\left(t_{n}\right), v_{0}\right), \quad \forall v=\left\{v_{0}, v_{b}\right\} \in S_{h}^{0}(j, \ell)
\end{aligned}
$$

\subsection{Properties of the bilinear form $a\left(u_{h}, v\right):[2]$}

Let $V$ be a Hilbert space with scalar product $(\cdot,)_{V},\left(V=H^{1}(D)\right)$, and corresponding norm $\|u\|_{H^{1}(D)}$. Suppose that $a\left(u_{h}, v\right)$ is a bilinear form on $V \times V$. We prove the properties of the bilinear form (V-elliptic and continuity).

Lemma 4.1 (continuity): A bilinear form defined on $V$ space equipped with norm \|\|$_{V}$ is continuous if there is a positive constant $C>0$, such that

$$
a\left(U_{h}^{n}, v\right) \leq C\left\|U_{h}^{n}\right\|_{1, D}\|v\|_{1, D} .
$$

Proof: By using Cauchy -Schwarz inequality in equation (16), we have

$$
\begin{aligned}
&\left|a\left(U_{h}^{n}, v\right)\right|=\left|\left(\lambda \nabla_{d} U_{h}^{n}, \nabla_{d} v\right)-\left(b U_{0}^{n}, \nabla_{d} v\right)\right| \\
& \leq|\lambda|_{L^{\infty}(K)}\left\|\nabla_{d} U_{h}^{n}\right\|_{L^{2}(D)}\left\|\nabla_{d} v\right\|_{L^{2}(D)}+|b|_{L^{\infty}(K)}\left\|U_{0}^{n}\right\|_{L^{2}(D)}\left\|\nabla_{d} v\right\|_{L^{2}(D)} \\
& \leq C_{1}\left\{\left\|\nabla_{d} U_{h}^{n}\right\|_{L^{2}(D)}\left\|\nabla_{d} v\right\|_{L^{2}(D)}+\left\|U_{0}^{n}\right\|_{L^{2}(D)}\left\|\nabla_{d} v\right\|_{L^{2}(D)}\right\} \\
& \leq C_{1} \sqrt{\left\|\nabla_{d} U_{h}^{n}\right\|_{L^{2}(D)}^{2}+\left\|U_{0}^{n}\right\|_{L^{2}(D)}^{2}} \times \sqrt{\left\|\nabla_{d} v\right\|_{L^{2}(D)}^{2}+\left\|\nabla_{d} v\right\|_{L^{2}(D)}^{2}} \\
& \leq C_{1} \sqrt{\left\|\nabla_{d} U_{h}^{n}\right\|_{L^{2}(D)}^{2}+\left\|U_{0}^{n}\right\|_{L^{2}(D)}^{2}} \times 2^{\frac{1}{2}} \sqrt{\left\|\nabla_{d} v\right\|_{L^{2}(D)}^{2}} \\
& \leq C\left\|U_{h}^{n}\right\|_{1, D}\|v\|_{1, D}
\end{aligned}
$$


Where, $C=\min \left\{|\lambda|_{L^{\infty}(K)},|b|_{L^{\infty}(K)}\right\}$.

Lemma 4.2 (V-elliptic): Let $U_{h}^{n}$ be the solution of equation (16) then there exist a positive constant $\alpha>0$,

$$
a\left(U_{h}^{n}, U_{h}^{n}\right) \geq \alpha\left\|U_{h}^{n}\right\|_{1, D}^{2}
$$

Proof: Let $\gamma=|\lambda|_{L^{\infty}(K)}$, be the $L^{\infty}$-norm of the coefficient $\lambda$, put $v=U_{h}^{n}$ in equation (16), we have

$$
a\left(U_{h}^{n}, U_{h}^{n}\right)=\left(\lambda \nabla_{d} U_{h}^{n}, \nabla_{d} U_{h}^{n}\right)-\left(b U_{0}^{n}, \nabla_{d} U_{h}^{n}\right)
$$

Now, by using Cauchy -Schwarz inequality

$$
\begin{aligned}
& \left|a\left(U_{h}^{n}, U_{h}^{n}\right)\right|=\left|\left(\lambda \nabla_{d} U_{h}^{n}, \nabla_{d} U_{h}^{n}\right)-\left(b U_{0}^{n}, \nabla_{d} U_{h}^{n}\right)\right|, \\
& \left|a\left(U_{h}^{n}, U_{h}^{n}\right)\right| \leq\left|\left(\lambda \nabla U_{h}^{n}, \nabla_{d} U_{h}^{n}\right)\right|+\left|\left(b U_{0}^{n}, \nabla_{d} U_{h}^{n}\right)\right|,
\end{aligned}
$$

by $\varepsilon$-inequality in the second term.

$$
\left|\left(b U_{0}^{n}, \nabla_{d} U_{h}^{n}\right)\right| \leq \frac{b^{2}}{2 \varepsilon}\left\|U_{0}^{n}\right\|_{L^{2}(D)}^{2}+\frac{\varepsilon}{2}\left\|\nabla_{d} U_{h}^{n}\right\|_{L^{2}(D)}^{2}
$$

Substituting (18) into (17), we have

$$
\begin{aligned}
\left|a\left(U_{h}^{n}, U_{h}^{n}\right)\right| & \geq \gamma\left\|\nabla_{d} U_{h}^{n}\right\|_{L^{2}(D)}^{2}+\frac{b^{2}}{2 \varepsilon}\left\|U_{0}^{n}\right\|_{L^{2}(D)}^{2}+\frac{\varepsilon}{2}\left\|\nabla_{d} U_{h}^{n}\right\|_{L^{2}(D)}^{2} \\
& \geq\left(\gamma+\frac{\varepsilon}{2}\right)\left\|\nabla_{d} U_{h}^{n}\right\|_{L^{2}(D)}^{2}+\frac{b^{2}}{2 \varepsilon}\left\|U_{0}^{n}\right\|_{L^{2}(D)}^{2} \\
& \geq \alpha\left(\left\|\nabla_{d} U_{h}^{n}\right\|_{L^{2}(D)}^{2}+\left\|U_{0}^{n}\right\|_{L^{2}(D)}^{2}\right) \\
& \geq \alpha\left\|U_{h}^{n}\right\|_{1, D}^{2}
\end{aligned}
$$

Where, $\alpha=\min \left\{\left(\gamma+\frac{\varepsilon}{2}\right), \frac{b^{2}}{2 \varepsilon}\right\}$

Theorem (stability): There exist a constant $\beta$ independent of $h$ such that,

$$
\left\|U_{h}^{N}\right\|_{L^{2}(D)}^{2}+k M \sum_{n=1}^{N}\left\|U_{0}^{n}\right\|_{L^{2}(D)}^{2} \leq k \sum_{n=1}^{N}\left\|f\left(t_{n}\right)\right\|_{L^{2}(D)}^{2}
$$

Proof: choose $v=U_{h}^{n}$ in equation (16), we gat

$$
\left(U_{h}^{n}, U_{h}^{n}\right)-\left(U_{h}^{n-1}, U_{h}^{n}\right)+k\left(\lambda \nabla_{d} U_{h}^{n}, \nabla_{d} U_{h}^{n}\right)-k\left(b U_{0}^{n}, \nabla_{d} U_{h}^{n}\right)=k\left(f\left(t_{n}\right), U_{0}^{n}\right)
$$

By Cauchy-Schwarz inequality and using the fact that of the first term, we have

$$
\left(U_{h}^{n}, U_{h}^{n}\right)-\left(U_{h}^{n-1}, U_{h}^{n}\right) \geq\left\|U_{h}^{n}\right\|_{L^{2}(D)}^{2}-\frac{1}{2}\left\|U_{h}^{n-1}\right\|_{L^{2}(D)}^{2}-\frac{1}{2}\left\|U_{h}^{n}\right\|_{L^{2}(D)}^{2}
$$

By the V-elliptic of the two terms, we have. 


$$
\left(\lambda \nabla_{d} U_{h}^{n}, \nabla_{d} U_{h}^{n}\right) \geq \alpha\left\|\nabla_{d} U_{h}^{n}\right\|_{L^{2}(D)}^{2}
$$

Using $\varepsilon$-inequality, we get

$$
\left(b U_{0}, \nabla_{d} U_{h}^{n}\right) \leq \frac{b^{2}}{2 \varepsilon}\left\|U_{0}^{n}\right\|_{L^{2}(D)}^{2}+\frac{\varepsilon}{2}\left\|\nabla_{d} U_{h}^{n}\right\|_{L^{2}(D)}^{2}
$$

Substituting (20), (21) and (22) in (19), we have

$$
\begin{gathered}
\left\|U_{h}^{n}\right\|_{L^{2}(D)}^{2}-\frac{1}{2}\left\|U_{h}^{n-1}\right\|_{L^{2}(D)}^{2}-\frac{1}{2}\left\|U_{h}^{n}\right\|+k \alpha\left\|\nabla_{d} U_{h}^{n}\right\|_{L^{2}(D)}^{2}+\frac{k b^{2}}{2 \varepsilon}\left\|U_{0}^{n}\right\|_{L^{2}(D)}^{2}+\frac{k \varepsilon}{2}\left\|\nabla_{d} U_{h}^{n}\right\|_{L^{2}(D)}^{2} \leq k\left\|f\left(t_{n}\right)\right\|\left\|U_{0}^{n}\right\| \\
\frac{1}{2}\left\|U_{h}^{n}\right\|_{L^{2}(D)}^{2}-\frac{1}{2}\left\|U_{h}^{n-1}\right\|+k \alpha\left\|\nabla_{d} U_{h}^{n}\right\|_{L^{2}(D)}^{2}+\frac{k b^{2}}{2 \varepsilon}\left\|U_{0}^{n}\right\|_{L^{2}(D)}^{2}+\frac{k \varepsilon}{2}\left\|\nabla_{d} U_{h}^{n}\right\|_{L^{2}(D)}^{2} \leq \frac{k}{2}\left\|U_{0}^{n}\right\|_{L^{2}(D)}^{2}+\frac{k}{2}\left\|f\left(t_{n}\right)\right\|_{L^{2}(D)}^{2} \\
\left\|U_{h}^{n}\right\|_{L^{2}(D)}^{2}-\left\|U_{h}^{n-1}\right\|_{L^{2}(D)}^{2}+k \beta\left\|\nabla_{d} U_{h}^{n}\right\|_{L^{2}(D)}^{2} \leq k\left\|U_{0}^{n}\right\|_{L^{2}(D)}^{2}+k\left\|f\left(t_{n}\right)\right\|_{L^{2}(D)}^{2}-k b^{2}\left\|U_{0}^{n}\right\|_{L^{2}(D)}^{2}
\end{gathered}
$$

Where $\beta=2 \alpha+\varepsilon \quad \varepsilon=1, \quad M=b^{2}-1$

$$
\left\|U_{h}^{n}\right\|_{L^{2}(D)}^{2}-\left\|U_{h}^{n-1}\right\|_{L^{2}(D)}^{2}+k M\left\|U_{0}^{n}\right\|_{L^{2}(D)}^{2} \leq k\left\|f\left(t_{n}\right)\right\|_{L^{2}(D)}^{2}
$$

So that by summation

$$
\left\|U_{h}^{N}\right\|_{L^{2}(D)}^{2}+k M \sum_{n=1}^{N}\left\|U_{0}^{n}\right\|_{L^{2}(D)}^{2} \leq k \sum_{n=1}^{N}\left\|f\left(t_{n}\right)\right\|_{L^{2}(D)}^{2} .
$$

\section{ENERGY CONSERVATION OF WEAK GALERKIN:}

Theorem (5.1): The numerical solution of equation (16) satisfies the energy conservation law.

$$
\int_{\Omega} \bar{\partial} U_{h}^{n} d \Omega=\int_{\Omega} f\left(t_{n}\right) d \Omega
$$

Proof: We chose a test function $v=\left\{v_{0}, v_{b}=0\right\}$ so that $v_{0}=1$ on $T$ and $v_{0}=0$ elsewhere. After integrating over $T$, we have

$$
\int_{T} \lambda \nabla_{d} U_{h}^{n} \nabla_{d} v d T=\int_{T} \lambda \nabla_{d} U_{h}^{n} \nabla_{d} 1 d T=0
$$

The second term of equation,

$$
\int_{T} b U_{0}^{n} \nabla_{d} v d T=\int_{T} b U_{0}^{n} \nabla_{d} 1 d T=0
$$

hence from equation (16) we get,

$$
\sum_{T \in T_{h} T} \int_{\partial} U_{h}^{n} d T=\sum_{T \in T_{h} T} \int_{f} f\left(t_{n}\right) d T
$$

Then,

$$
\int_{\Omega}^{\bar{\partial}} U_{h}^{n} d \Omega=\int_{\Omega} f\left(t_{n}\right) d \Omega
$$

\section{Error analysis for Full- discrete weak Galerkin Finite Element Method:}

In this section we show that the theoretical analysis of error between the exact solution of equation (3) and the weak Galerkin approximation (16) by using the $L^{2}$-projection $Q_{h} u$ of the exact solution and $\pi_{h}$ be the usual projection define in (13) 
Lemma 6.1: For $u \in H^{1+r}(\Omega)$ with $r>0$. We have

$$
\left\|\pi_{h}(\lambda \nabla u)-\lambda \nabla_{d}\left(Q_{h} u\right)\right\|_{L^{2}(D)} \leq C h^{1+r}\|u\|_{1+r}
$$

Proof: since from (11) we have $\nabla_{d}\left(Q_{h} u\right)=R_{h}(\nabla u)$, then

$$
\left\|\pi_{h}(\lambda \nabla u)-\lambda \nabla_{d}\left(Q_{h} u\right)\right\|_{L^{2}(D)}=\left\|\pi_{h}(\lambda \nabla u)-\lambda R_{h}(\nabla u)\right\|_{L^{2}(D)}
$$

Now, addition and subtraction $(\lambda \nabla u)$ and by using the triangle inequality we have

$$
\begin{aligned}
\left\|\pi_{h}(\lambda \nabla u)-\lambda \nabla u+\lambda \nabla u-\lambda R(\nabla u)\right\|_{L^{2}(D)} & \leq\left\|\pi_{h}(\lambda \nabla u)-\lambda \nabla u\right\|_{L^{2}(D)}+\|\lambda \nabla u-\lambda R(\nabla u)\|_{L^{2}(D)} \\
& \leq C_{1} h^{r}\|\nabla u\|_{L^{2}(D)}+C_{2} h^{r}\|\nabla u\|_{L^{2}(D)} \\
& \leq C h^{r+1}\|u\|_{1+r}
\end{aligned}
$$

Theorem 9.1: Let $u \in H^{1+r}(\Omega)$ and $u$ be the solution of (15) and $U_{h}^{n}$ be the weak Galerkin approximation of equation (16). Then there exists a constant $C$ such that.

$$
\left\|U_{h}^{n}-u^{n}\right\|_{L^{2}(D)} \leq C\left(h^{1+r}\|u\|_{1+r}+k \int_{0}^{t_{n}}\left\|u_{t t}\right\|_{L^{2}(D)} d s\right)
$$

Proof: since $u\left(t_{n}\right)$ satisfies, $u\left(t_{n}\right)=u^{n}$,

Let

$$
\begin{aligned}
& U_{h}^{n}-u^{n}=U_{h}^{n}-Q_{h} u^{n}+Q_{h} u^{n}-u^{n} \\
& U_{h}^{n}-u^{n}=\theta^{n}+\rho^{n}
\end{aligned}
$$

Where, $\rho^{n}=Q_{h} u^{n}-u^{n}, \quad \theta^{n}=U_{h}^{n}-Q_{h} u^{n}$

$$
\left\|U_{h}^{n}-u^{n}\right\|_{L^{2}(D)} \leq\left\|\theta^{n}\right\|_{L^{2}(D)}+\left\|\rho^{n}\right\|_{L^{2}(D)}
$$

From [19] we have

$$
\left\|\rho^{n}\right\|_{L^{2}(D)} \leq C h^{r+1}\|u\|_{1+r}
$$

To estimate $\theta$, from equation (15) and lemma (3.2.2), we have

$$
\left(\bar{\partial} Q_{h} u^{n}, v_{0}\right)+\left(\pi_{h}\left(\lambda \nabla u^{n}\right), \nabla_{d} v\right)-\left(\pi_{h}\left(b u^{n}\right), \nabla_{d} v\right)=\left(f\left(t_{n}\right), v_{0}\right) .
$$

Now, addition and subtraction $a\left(Q_{h} u^{n}, v\right)=\left(\lambda \nabla_{d}\left(Q_{h} u^{n}\right), \nabla_{d} v\right)-\left(b\left(Q_{0} u^{n}\right), \nabla_{d} v\right)$ on the left hand side of the above equation and then using (13), we have

$$
\begin{gathered}
\left(\bar{\partial} Q_{h} u^{n}, v_{0}\right)+\left(\lambda \nabla_{d}\left(Q_{h} u^{n}\right), \nabla_{d} v\right)-\left(b\left(Q_{0} u^{n}\right), \nabla_{d} v\right)+ \\
\left(\pi_{h}\left(\lambda \nabla u^{n}\right)-\lambda R_{h}\left(\nabla u^{n}\right), \nabla_{d} v\right)-\left(\pi_{h}\left(b u^{n}\right)-b\left(Q_{0} u^{n}\right), \nabla_{d} v\right)=\left(f\left(t_{n}\right), v_{0}\right)
\end{gathered}
$$

Combining equation (16) with the above equation, we have

$$
\begin{gathered}
\bar{\partial}\left(U_{h}^{n}-Q_{h} u^{n}, v_{0}\right)+\left(\lambda \nabla_{d}\left(U_{h}^{n}-Q_{h} u^{n}\right), \nabla_{d} v\right)-\left(b\left(U_{0}^{n}-Q_{h} u^{n}\right), \nabla_{d} v\right)= \\
\left(u_{t}^{n}-\bar{\partial} u^{n}, v_{0}\right)+\left(\pi_{h}\left(\lambda \nabla u^{n}\right)-\lambda R_{h}\left(\nabla u^{n}\right), \nabla_{d} v\right)-\left(\pi_{h}\left(b u^{n}\right)-b\left(Q_{0} u^{n}\right), \nabla_{d} v\right)
\end{gathered}
$$

Since $\theta^{n}=U_{h}^{n}-Q_{h} u^{n}$, we have

$$
\bar{\partial}\left(\theta^{n}, v_{0}\right)+\left(\lambda \nabla_{d} \theta^{n}, \nabla_{d} v\right)-\left(b\left(U_{0}^{n}-Q_{h} u^{n}\right), \nabla_{d} v\right)=\left(u_{t}^{n}-\bar{\partial} u^{n}, v_{0}\right)+
$$




$$
\left(\pi_{h}\left(\lambda \nabla u^{n}\right)-\lambda R_{h}\left(\nabla u^{n}\right), \nabla_{d} v\right)-\left(\pi_{h}\left(b u^{n}\right)-b\left(Q_{0} u^{n}\right), \nabla_{d} v\right)
$$

Use $v=\theta^{n}$ in the error equation, we obtain

$$
\begin{aligned}
& \quad \bar{\partial}\left(\theta^{n}, \theta^{n}\right)+\left(\lambda \nabla_{d} \theta^{n}, \nabla_{d} \theta^{n}\right)-\left(b\left(U_{0}^{n}-Q_{h} u^{n}\right), \nabla_{d} \theta^{n}\right)=\left(u_{t}^{n}-\bar{\partial} u^{n}, \theta^{n}\right) \\
& +\left(\pi_{h}\left(\lambda \nabla u^{n}\right)-\lambda R_{h}\left(\nabla u^{n}\right), \nabla_{d} \theta^{n}\right)-\left(\pi_{h}\left(b u^{n}\right)-b\left(Q_{0} u^{n}\right), \nabla_{d} \theta^{n}\right)
\end{aligned}
$$

Let

$$
\begin{aligned}
& I_{1}^{n}=u_{t}^{n}-\bar{\partial} u^{n}, \\
& I_{2}^{n}=\pi_{h}\left(\lambda \nabla u^{n}\right)-\lambda R_{h}\left(\nabla u^{n}\right), \\
& I_{3}^{n}=\pi_{h}\left(b u^{n}\right)-b\left(Q_{0} u^{n}\right) .
\end{aligned}
$$

We have

$$
\begin{gathered}
\bar{\partial}\left(\theta^{n}, \theta^{n}\right)+\left(\lambda \nabla_{d} \theta^{n}, \nabla_{d} \theta^{n}\right)-\left(b\left(U_{0}^{n}-Q_{h} u^{n}\right), \nabla_{d} \theta^{n}\right)= \\
\left(I_{1}^{n}, \theta^{n}\right)+\left(I_{2}^{n}, \nabla_{d} \theta^{n}\right)+\left(I_{3}^{n}, \nabla_{d} \theta^{n}\right) \\
\left(\frac{\left(\theta^{n}, \theta^{n-1}\right)}{k}, \theta^{n}\right)+\left(\lambda \nabla_{d} \theta^{n}, \nabla_{d} \theta^{n}\right)-\left(b\left(U_{0}^{n}-Q_{h} u^{n}\right), \nabla_{d} \theta^{n}\right)= \\
\left(I_{1}^{n}, \theta^{n}\right)+\left(I_{2}^{n}, \nabla_{d} \theta^{n}\right)+\left(I_{3}^{n}, \nabla_{d} \theta^{n}\right) \\
\left(\frac{\left(\theta^{n}, \theta^{n-1}\right)}{k}, \theta^{n}\right)+a\left(\theta^{n}, \theta^{n}\right)=\left(I_{1}^{n}, \theta^{n}\right)+\left(I_{2}^{n}, \nabla_{d} \theta^{n}\right)+\left(I_{3}^{n}, \nabla_{d} \theta^{n}\right)
\end{gathered}
$$

By V-elliptic of the bilinear form and Cauchy-Schwarz inequality and $\varepsilon$-inequality, we obtain

$$
\begin{aligned}
& \left\|\theta^{n}\right\|_{L^{2}(D)}^{2}-\left(\theta^{n-1}, \theta^{n}\right)+\alpha k\left\|\nabla_{d} \theta^{n}\right\|_{L^{2}(D)}^{2}=k\left\|I_{1}^{n}\right\|_{L^{2}(D)}\left\|\theta^{n}\right\|_{L^{2}(D)} \\
& \quad+k\left\|I_{2}^{n}\right\|_{L^{2}(D)}\left\|\nabla_{d} \theta^{n}\right\|_{L^{2}(D)}+k\left\|I_{3}^{n}\right\|_{L^{2}(D)}\left\|\nabla_{d} \theta^{n}\right\|_{L^{2}(D)}
\end{aligned}
$$

Using here the fact that.

$$
\begin{aligned}
& \left\|\theta^{n}\right\|_{L^{2}(D)}^{2}+\alpha k\left\|\nabla_{d} \theta^{n}\right\|_{L^{2}(D)}^{2}-\frac{1}{2}\left\|\theta^{n-1}\right\|_{L^{2}(D)}^{2}-\frac{1}{2}\left\|\theta^{n}\right\|_{L^{2}(D)}^{2} \\
& \leq k\left\|I_{1}^{n}\right\|_{L^{2}(D)}\left\|\theta^{n}\right\|_{L^{2}(D)}+k\left\|I_{2}^{n}\right\|_{L^{2}(D)}\left\|\nabla_{d} \theta^{n}\right\|_{L^{2}(D)}+k\left\|I_{3}^{n}\right\|_{L^{2}(D)}\left\|\nabla_{d} \theta^{n}\right\|_{L^{2}(D)}
\end{aligned}
$$

By the Poincare inequality of Lemma [14], we have

$$
\frac{1}{2}\left\|\theta^{n}\right\|_{L^{2}(D)}^{2}-\frac{1}{2}\left\|\theta^{n-1}\right\|_{L^{2}(D)}^{2}+\alpha k\left\|\nabla_{d} \theta^{n}\right\|_{L^{2}(D)}^{2} \leq k\left(C\left\|I_{1}^{n}\right\|_{L^{2}(D)}+\left\|I_{2}^{n}\right\|_{L^{2}(D)}+\left\|I_{3}^{n}\right\|_{L^{2}(D)}\right)\left\|\nabla_{d} \theta^{n}\right\|_{L^{2}(D)}
$$

Then by $\varepsilon$-inequality on the right hand side of the above equation, it follows

$$
\begin{gathered}
\frac{1}{2}\left\|\theta^{n}\right\|_{L^{2}(D)}^{2}-\frac{1}{2}\left\|\theta^{n-1}\right\|_{L^{2}(D)}^{2}+\alpha k\left\|\nabla_{d} \theta^{n}\right\|_{L^{2}(D)}^{2} \leq \\
\frac{k}{2 \alpha}\left(C\left\|I_{1}^{n}\right\|_{L^{2}(D)}+\left\|I_{2}^{n}\right\|_{L^{2}(D)}+\left\|I_{3}^{n}\right\|_{L^{2}(D)}\right)^{2}+\frac{\alpha k}{2}\left\|\nabla_{d} \theta^{n}\right\|_{L^{2}(D)}^{2} \\
\frac{1}{2}\left\|\theta^{n}\right\|_{L^{2}(D)}^{2}-\frac{1}{2}\left\|\theta^{n-1}\right\|_{L^{2}(D)}^{2}+\frac{\alpha k}{2}\left\|\nabla_{d} \theta^{n}\right\|_{L^{2}(D)}^{2} \leq \frac{k}{2 \alpha}\left(C\left\|I_{1}^{n}\right\|_{L^{2}(D)}+\left\|I_{2}^{n}\right\|_{L^{2}(D)}+\left\|I_{3}^{n}\right\|_{L^{2}(D)}\right)^{2}
\end{gathered}
$$

So that 


$$
\begin{aligned}
& \left\|\theta^{n}\right\|_{L^{2}(D)}^{2}-\left\|\theta^{n-1}\right\|_{L^{2}(D)}^{2}+\alpha k\left\|\nabla_{d} \theta^{n}\right\|_{L^{2}(D)}^{2} \leq \frac{k C}{\alpha}\left\|I_{1}^{n}\right\|_{L^{2}(D)}^{2}+\frac{k}{\alpha}\left\|I_{2}^{n}\right\|_{L^{2}(D)}^{2}+\frac{k}{\alpha}\left\|I_{3}^{n}\right\|_{L^{2}(D)}^{2} \\
& \left\|\theta^{n}\right\|_{L^{2}(D)}^{2}-\left\|\theta^{n-1}\right\|_{L^{2}(D)}^{2} \leq \frac{k C}{\alpha}\left\|I_{1}^{n}\right\|_{L^{2}(D)}^{2}+\frac{k}{\alpha}\left\|I_{2}^{n}\right\|_{L^{2}(D)}^{2}+\frac{k}{\alpha}\left\|I_{3}^{n}\right\|_{L^{2}(D)}^{2}
\end{aligned}
$$

And, by repeated application,

$$
\left\|\theta^{n}\right\|_{L^{2}(D)}^{2} \leq \frac{k C}{\alpha} \sum_{j=1}^{n}\left\|I_{1}^{j}\right\|_{L^{2}(D)}^{2}+\frac{k}{\alpha} \sum_{j=1}^{n}\left\|I_{2}^{j}\right\|_{L^{2}(D)}^{2}+\frac{k}{\alpha} \sum_{j=1}^{n}\left\|I_{3}^{j}\right\|_{L^{2}(D)}^{2}
$$

The first term for the equation (26)

$$
\begin{aligned}
k I_{1}^{j} & =k u_{t}^{j}-\left(u^{j}-u^{j-1}\right) \\
& =k u_{t}^{j}-\int_{t_{j-1}}^{t_{j}} u_{t}(s) d s \\
& =\left(t_{j}-t_{j-1}\right) u_{t}^{j}-\int_{t_{j-1}}^{t_{j}} u_{t}(s) d s
\end{aligned}
$$

Adding and subtracting $t_{j-1} u_{t}^{j-1}$, we get

$$
\begin{aligned}
& =t_{j} u_{t}^{j}-t_{j-1} u_{t}^{j-1}-\left(t_{j-1} u_{t}^{j}-t_{j-1} u_{t}^{j-1}\right)-\int_{t_{j-1}}^{t_{j}} u_{t}(s) d s \\
& =\int_{t_{j-1}}^{t_{j}} s u_{t t}(s) d s-t_{j-1} \int_{t_{j-1}}^{t_{j}} u_{t t}(s) d s \\
k I_{1}^{j} & =\int_{t_{j-1}}^{t_{j}}\left(s-t_{j-1}\right) u_{t t}(s) d s \\
I_{1}^{j} & =\frac{1}{k} \int_{t_{j-1}}^{t_{j}}\left(s-t_{j-1}\right) u_{t t}(s) d s
\end{aligned}
$$

So that

$$
\begin{aligned}
& \left\|I_{1}^{j}\right\|_{L^{2}(D)}^{2}=\int_{\Omega}\left\{\frac{1}{k} \int_{t_{n-1}}^{t_{n}}\left(s-t_{n-1}\right) u_{t t}(s) d s\right\}^{2} d \Omega \\
& \quad \leq \frac{1}{k^{2}} \int_{\Omega}\left[\int_{t_{n-1}}^{t_{n}}\left(s-t_{n-1}\right)^{2} d s \int_{t_{n-1}}^{t_{n}} u_{t t}^{2}(s) d s\right] d \Omega \\
& \quad \leq C k \int_{t_{n-1}}^{t_{n}}\left\|u_{t t}\right\|_{L^{2}(D)}^{2} d s .
\end{aligned}
$$

To approximate $I_{2}^{j}$, since $\nabla_{d}\left(Q_{h} u\right)=R_{h}(\nabla u)$ and $u \in H^{1+r}(\Omega)$, we get

$$
I_{2}^{j}=\left\|\pi_{h}\left(\lambda \nabla u^{n}\right)-\lambda R_{h}\left(\nabla u^{n}\right)\right\|_{L^{2}(D)}^{2}
$$


Addition and subtraction $\left(\lambda \nabla u^{n}\right)$ and by using the triangle inequality, we have

$$
\begin{aligned}
& \leq\left\|\pi_{h}\left(\lambda \nabla u^{n}\right)-\lambda \nabla u^{n}\right\|_{L^{2}(D)}^{2}+\left\|\lambda \nabla u^{n}-\lambda R_{h}\left(\nabla u^{n}\right)\right\|_{L^{2}(D)}^{2} \\
& \leq\left\|\pi_{h}\left(\lambda \nabla u^{n}\right)-\lambda \nabla u^{n}\right\|_{L^{2}(D)}^{2}+\left\|\lambda \nabla u^{n}-\nabla_{d}\left(Q_{h} u^{n}\right)\right\|_{L^{2}(D)}^{2} \\
& \leq C h^{2 r+2}\|u\|_{1+r}^{2}
\end{aligned}
$$

To approximate, $I_{3}^{j}$ and $Q_{0} u^{n}=u^{n}$

$$
\begin{aligned}
I_{3}^{j} & =\left\|\pi_{h}\left(b u^{n}\right)-b\left(Q_{0} u^{n}\right)\right\|_{L^{2}(D)}^{2} \\
& =\left\|\pi_{h}\left(b u^{n}\right)-b u^{n}\right\|_{L^{2}(D)}^{2} \\
& \leq C h^{2 r+2}\|u\|_{1+r}^{2}
\end{aligned}
$$

Substitute (27), (28), (29) into (26), we have

$$
\left\|\theta^{n}\right\|_{L^{2}(D)}^{2} \leq C\left(h^{2 r+2}\|u\|_{1+r}^{2}+k^{2} \int_{0}^{t_{n}}\left\|u_{t t}\right\|_{L^{2}(D)}^{2} d s\right) .
$$

And in particular

$$
\left\|\theta^{n}\right\|_{L^{2}(D)} \leq C\left(h^{r+1}\|u\|_{1+r}+k \int_{0}^{t_{n}}\left\|u_{t t}\right\|_{L^{2}(D)} d s\right)
$$

Then by substituting (30),(24) into(23),we get

$$
\left\|U_{h}^{n}-u^{n}\right\|_{L^{2}(D)} \leq C\left(h^{1+r}\|u\|_{1+r}+k \int_{0}^{t_{n}}\left\|u_{t t}\right\|_{L^{2}(D)} d s\right)
$$

\section{Conclusion:}

The present paper presents the full- discrete weak Galerkin finite element method for the non-steady diffusion-convection problem. by using this method we proved the energy conservation law to verified that the numerical flux to be continuous a cross the edge of each element $K$ through a selection of the test function $v=\left\{v_{0}, v_{b}\right\}$.We have proved the properties of the bilinear form $a(u, v)$, (v-elliptic and continuity ),stability, also we prove the convergence of the scheme.

\section{REFERENCES}

[1] C. Jan and Miloslav Feistauer, Theory of the space-time discontinuous Galerkin method for nonstationary parabolic problems with nonlinear convection and diffusion, Mathematical Institute of the Charles University,(2011),pp.1-20.

[2] C. Johnson, Numerical solution of partial differential equations by the finite element method, Cambridge University Press,(1987).

[3] Hashim A. Kashkool, Yahea H. Saleem and Ghazi A. Muften, Error estimate of discontinuous Galerkin finite element method for space time discretization of Convection -Diffusion problem, IJPRET,2013; Volume 2(4):25-50.

[4] Hashim A. Kashkool, Upwind type finite element method for nonlinear Convection

-Diffusion problem and applications to numerical reservoir simulation, school of Mathematical Sciences, Nankai Universty, China (2002).

[5] J.T. Oden, and T.N. Reddy, An introduction to mathematical theory of finite element, John Wiley and Sons Inc., (1976)

[6] J. Wang and X.Ye, A weak Galerkin finite element method for second-order elliptic problems, arXiv:1104.2897v1,(2011). 
[7] K. Baba and M. Tabata, On a conservation upwind finite element scheme for convection- diffution equation, R.A.I.R.O., Numerical analysis, 15, no. 1, pp.3-25, (1981).

[8] L. Mu, J. Wang, X. Ye, Weak Galerkin finite element method on polytopal meshes, arXiv:1204.3655.

[9] L. Mu, J. Wang, X. Ye, A weak Galerkin finite element method with polynomial reduction, arXiv:1304.6481v1,(2013).

[10] Mu, L., Wang, J., Wei, G., Ye., X., Zhao, S., A weak Galerkin finite element method for the elliptic interface problem, arXiv:1201.6438v2,(2012).

[11] Mu, L., Wang, J., Wang, Y., Ye., X., A weak Galerkin mixed finite element method for Biharmonic equations, arXiv:1210.3818,(2012).

[12] Mu, L., Wang, J., Ye., X., Zhang, S., A $C^{0}$-weak Galerkin finite element method for the biharmonic equation, arXiv:1212.0250,(2012)

[13] Mu, L., Wang, J., Ye., X., Zhao, S., Numerical studies on the weak Galerkin method for Helmholtz equation with large wave number, arXiv:1111.0671,(2011).

[14] P. G. Ciarlet, The finite element method for elliptic problems, Amesterdam. North Holland, 1978.

[15] Qiaoluan H. LI and J. Wang, Weak Galerkin finite element methods for parabolic equations, arXiv:1212.3637v2.(2013).

[16] Quarteroni and A.valli, Numerical approximation of partial differential equations, ISBN 3-540-5711-6, Springer-Verlag Berlin Heidelbery-NewYourk,(1997).

[17] S. Sun and M. F. Wheeler, Symmetric and nonsymmetric discontinuous Galerkin methods for reactive transport in porous media. SIAM J. Numér. Anal. 43 (2005), 195-219.

[18] Uwe Risch, An upwind finite element method for singularly perturbed

elliptic problems and local estimates in the Lø-norm, MAN, 34, no. 2, pp. 235-264, (1990).

[19] V.Thomee, Galerkin finite element for parabolic problem, Springer-Verlag, Berlin,1054,(1984)

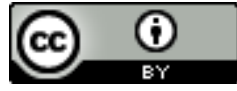

This work is licensed under a Creative Commons Attribution 4.0 International License. 